\title{
Face Recognition System: Alignment And Illumination Using Sparse Representation
}

\author{
Prof.A.S. Deshpande ${ }^{1}$, Vinayak Karkud ${ }^{2}$, Amol Kokare ${ }^{3}$, Rohit Aurade $^{4}$ \\ Imperial College Of Engineering \& Research, Wagholi, Pune $e^{1,2,3,4}$
}

\begin{abstract}
: face recognition algorithms work on public data sets, but it degrade sharply when they are used in a real recognition system. This is due to the difficulty of illumination, image misalignment in the test image. We consider a fact where the input images are well controlled and test images are only low controlled. We propose a proper simple face recognition system that runs against a high degree of robustness and illumination variation, image misalignment. The system uses tools from sparse representation to align a test face image to a set of database images. We determine how to capture a set of database images with some illumination variation that they span test images taken under illumination. In order to find how our algorithms work under practical testing conditions, we have evaluate a complete face recognition system. Our system can efficiently and effectively recognize faces under different conditions.
\end{abstract}

Keywords: NN, NS, LDA, SVM

\section{INTRODUCTION}

The Face recognition applications having two categories i.e. illumination and alignment. Face recognition has recently achieve a lot of success in a family of lessvaluable applications such as online image search and family photo album organization. At the other end, there are the terrorist watch list and mass surveillance applications that have, for the most part, the field of face recognition research. There are many face recognition applications that fall between these last, where best recognition performance is require. These applications include access control for secure facilities (e.g., company and office, computer systems, automobiles teller machines).

These applications are very interesting due to their sociological impact. Since the gallery subjects are allies, rather than opponents, of the recognition system, this creates the possibility of carefully controlling the acquisition of the training data. While the same can be said for other biometrics such as fingerprints and iris recognition, face recognition has the potential of working with test data that are much low controlled, allowing the access control system to be made less intrusive to the users of the system. To some extent, the goal of this paper is to show how a reliable face recognition system can be built for this restricted, but still important, scenario. Very few recognition systems specifically focus on the applications where many well-controlled database images are available. For these, the classical face recognition methods, are well known for their speed and simplicity, as well as they effectively work on illumination models. Also, their performance has been shown to be extremely better not only to alignment variation, but to illumination.

\section{PROBLEM STATEMENT}

To build and evaluate a face recognition model with robustness against Common facial occlusions (glasses, mask,..), Face pose estimation, Face scale variation.

\section{LITERATURE REVIEW}

To determine effectiveness of our alignment algorithm, we compare our result to baseline linearprojection-based algorithms, such as Nearest Neighbor (NN), Nearest Subspace (NS), and Linear Discriminate Analysis (LDA). Since these algorithms requires pixelaccurate alignment, they are not work well if the test image is not well aligned with the database images.

Nearest Neighbor algorithm is a non-parametric method used for classification and regression. The nonparametric technique which means the technique which do not relying on data belonging to any particular distribution. The disadvantage of this algorithm are Image dimension reduction is required and the class-outlier with $\mathrm{K}-\mathrm{NN}$ produce noise.

Nearest Subspace algorithm the linear and affine subspace are commonly used to describe the appearance of object under different lighting, viewpoint etc. A natural problem arising is a given image portion represent as a point in some high dimensional space. Nearest subspace can be located significantly faster than the exact nearest subspace while at same time it find better matches. We know that no reduction to points can be done without an offset, but this does not rule out other approaches to solve the problem of high dimensional space.

The paper, proposes a face recognition method for locate the problem of unconstrained face recognition from remotely acquired images. The main factors to affect this system is challenging the image degradation due to blur, appearance variations due to illumination and pose. We have proposed a system for recognizing human faces from images taken under practical conditions that is conceptually simple, well motivated, and competitive with state-of-the-art recognition systems for access control scenarios. 


\section{BLOCK DIAGRAM}

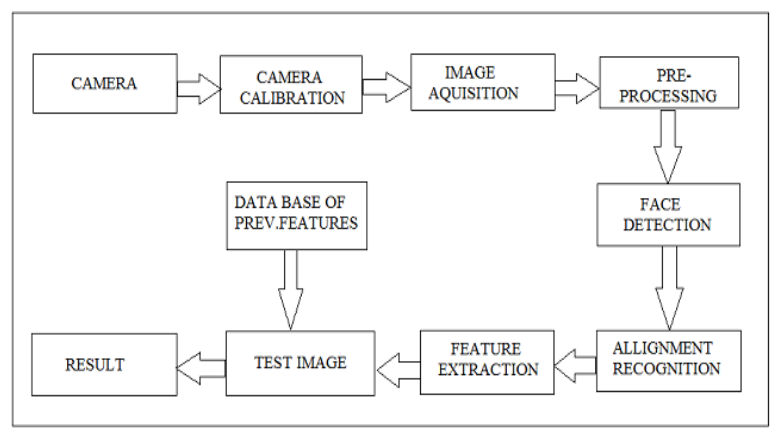

\section{ALGORITHM}

\section{I) Viola Jones}

\section{Viola jones technique overview:}

The main principle of the Viola-Jones algorithm is first to scan a sub-window capable of detecting faces across a given input image. The proper image processing aim would be to rescale the input image to different sizes and then run the fix size detector through these database images. This aim becomes time consuming due to the calculation of the different size images. The other option is to the standard approach Viola-Jones detector instead of the input image and run the detector many times through the image - each time with a different size. At first one might clear that both approaches to be equally time consuming, but Viola-Jones have advantages of a scale invariable detector that requires the same number of calculations and its does not depend on the size.

Three major phases of the algorithm :

1.Feature extraction

2.Classification using boosting

3. Multi-scale detection algorithm

In the feature extraction and feature valuation we used rectangular features, with a new image and rescale the image so the speed of the calculation is increased due to which the calculations are reduced. A mixer of the basic classifiers is very efficent. The features of the given image are easy to calculate using viola- jones technique, here the white areas are subtracted from the black ones, the main representation of the image called the integral image this makes feature extraction faster and easier.

\section{Feature Extraction}

Features are extracted from given sample image using sub windows. The standard size for a sub window is 24 by 24 pixels are used. All four feature types are scaled and shifted across all possible combinations. Here we can take the features like distance between eyes, nose \& mouth, eyes \& nose. These features are placed in the form of integer values. For comparison of images and for less time consumption we will not save hole image but we are saving features of images that will reduce the size.

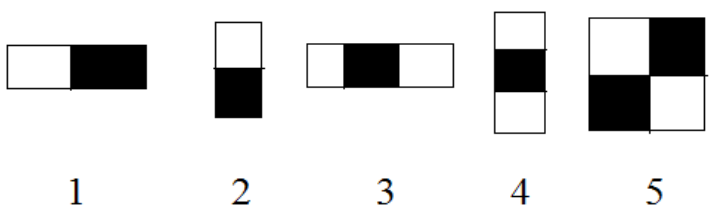

Fig: Different models of Features

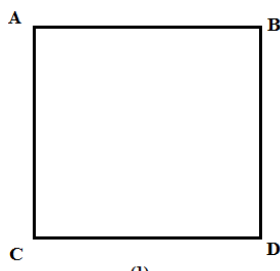

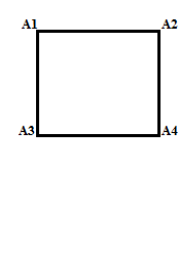

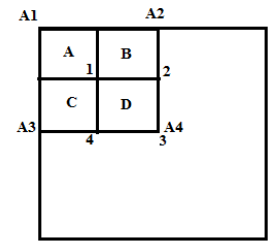

Fig: (1)main image (2)focused area (3)integrated image

The equations that are used to find the sum of pixels within rectangle $\mathrm{D}$ can be obtained with four array references. The value of integral image at position 1 is the sum of pixels in the rectangle $A$. The value at position 2 is $[\mathrm{A}+\mathrm{B}]$, at position 3 is $[\mathrm{A}+\mathrm{C}]$, and at position 4 is $[A+B+C+D]$, the sum within $D$ can be computed as $[4+1-$ $(2+3)]$.

\section{II) Support Vector Machine (SVM)}

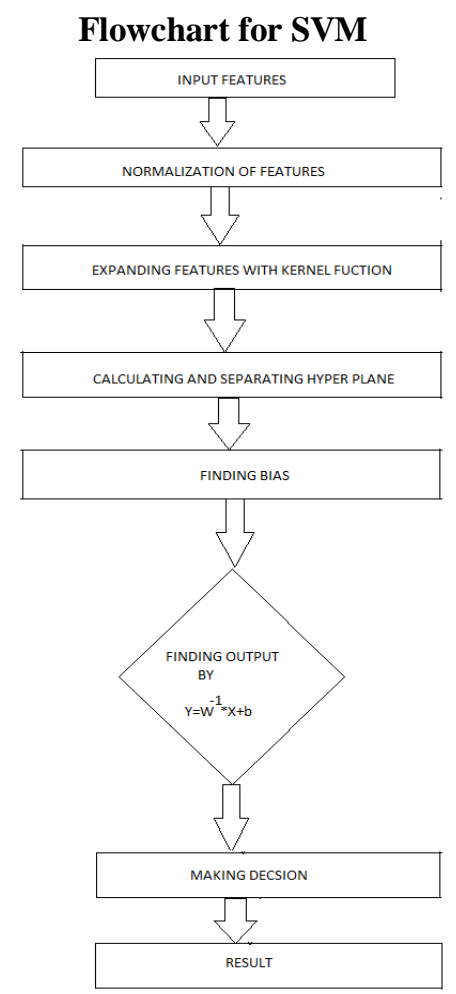


Support Vector Machine (SVM) is a supervised learning algorithm developed by Vladimir Vapnik and it was first used in 1992, introduced by Vapnik, Boser and Guyon in COLT-92 (it is said that Vladimir Vapnik has tells its idea in 1979 in his paper but its main development was in the 90's). Before SVM for many years Neural Networks was the main champion, it was the most effective learning algorithm. SVM became popular because of its success in handwritten digit recognition (in NIST (1998)). It gave best accuracy that is comparable to vitiate and carefully constructed neural networks with studied features in a handwriting recognition task. Much more effective "off the shelf " algorithm than Neural Networks : It generalize good on hide data and is easier to operate and does not have any relation in contrast to neural networks that may have many local optima and takes more time to converge.

SVM has successful applications in many complicated, real-world problems such as text and image classification, hand-writing recognition, data mining and even stock market! In many of these applications SVM is the best algorithm.

We are given a set of $\mathrm{n}$ points (vectors) : such that is a vector of length $\mathrm{m}$, and each belong to one of two classes we label them by " +1 " and " -1 ". our training set is:

$$
(x 1, y 1),(x 2, y 2), \ldots . .(x n, y n)
$$

We want to find a separating hyperplane $(w \cdot x+b=0)$ that separates these points into the two classes. "The positives" (class "+1") and "The negatives" (class "-1"). (Assuming that they are linearly separable)

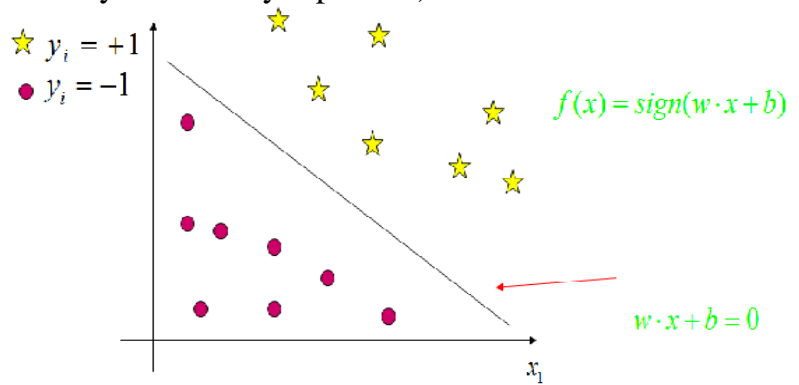

Fig: Separating hyperplane

There are many possible separating hyperplanes shown in below fig.

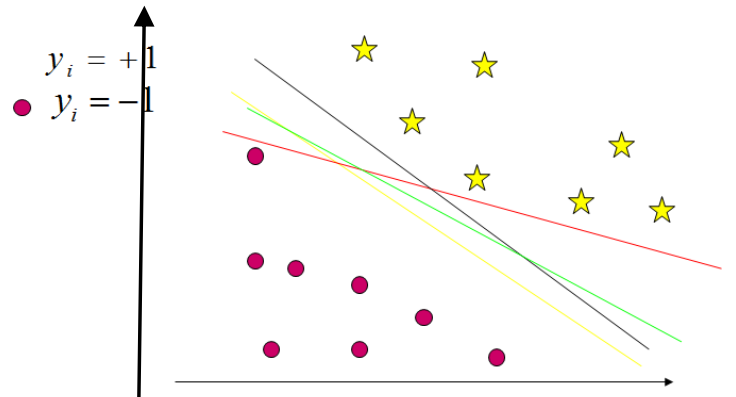

Fig: All possible hyperplanes
Hyperplane should be as far as possible from any sample point. This way a new data that is close to the old samples will be classified correctly.

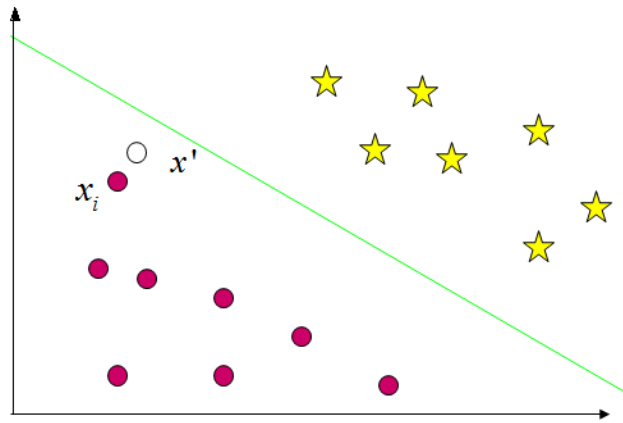

Fig: Good generalization

\section{CONCLUSION}

The aim of this paper is to overcome the problems occurred in the normal face recognition system like proper alignment, illumination, angle. The system achieves the stable performance under a wide range of variations in illumination, misalignment, and even under small amounts of pose.

It is possible to overcome the problem using Viola-Jones algorithm and Support Vector Machine classifier we able

So to increase the efficiency and reduce the drawbacks. It is not possible $\mathrm{t}$ the drawbacks. It is not possible to get accurate recognition of face in improper face area of the human face and also some effect present due to distance of face from camera. But overall we recognize the face very effectively.

\section{REFERENCES}

[1] M. Turk and A. Pentland, "Eigenfaces for Recognition," Proc. IEEE Conf. Computer Vision and Pattern Recognition, 1991.

[2] P. Belhumeur, J. Hespanda, and D. Kriegman, "Eigenfaces versus Fisherfaces: Recognition Using Class Specific Linear Projection, IEEE Trans. Pattern Analysis and Machine Intelligence, vol. 19, no. 7, pp. 711 720, July 1997.

[3] J. Wright, A. Yang, A. Ganesh, S. Sastry, and Y. Ma, "Robust Face Recognition via Sparse Representation," IEEE Trans. Pattern Analysis and Machine Intelligence, vol. 31, no. 2, pp. 210-227, Feb. 2009

[4] B. Amberg, A. Blake, A. Fitzgibbon, S. Romdhani, and T. Vetter, "Reconstructing High Quality Face-Surfaces Using Model Based Stereo,” Proc. IEEE Int'l Conf. Computer Vision, pp. 1-8, 2007.

[5] V. Blanz and T. Vetter, "Face Recognition Based on Fitting a 3D Morphable Model," IEEE Trans. Pattern Analysis and Machine Intelligence, vol. 25, no. 9, pp. 1063-1074, Sept. 2003.

[6] T. Cootes, G. Edwards, and C. Taylor, "Active Appearance Models," IEEE Trans. Pattern Analysis and Machine Intelligence, vol. 23, no. 6, pp. 681-685, June 2001

[7] T. Cootes and C. Taylor, "Active Shape Models- 'Smart Snakes'," Proc. British Machine Vision Conf., 1992.

[8] B. Lucas and T. Kanade, "An Iterative Image Registration Technique with an Application to Stereo Vision," Proc. Int'l Joint Conf. Artificial Intelligence, vol. 3, pp. 674-679, 1981.

[9] P. Belhumeur and G. Hager, "Tracking in 3D: Image Variability Decomposition for Recovering Object Pose and Illumination," Pattern Analysis and Applications, vol. 2, pp. 82-91, 1999.

[10] H. Murase and S. Nayar, "Visual Learning and Recognition of 3D Objects from Appearance," Int'l J. Computer Vision, vol. 14, pp. 5-24, 1995. 
International Journal of Advanced Research in Computer and Communication Engineering Vol. 4, Issue 3, March 2015

\section{BIOGRAPHIES}

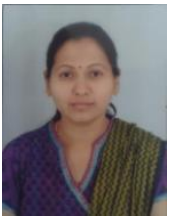

Prof. Anuradha S. Deshpande, BE ECE, ME Electronics. She is an Assistant Professor at JSPM's ICOER. She published "DWT based satellite color image resolution enhancement" Vol. 3 Issue 2 in IJMER

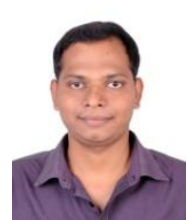

Vinayak Rohidas Karkud, Student of BE ECE at JSPM's ICOER, Pune.

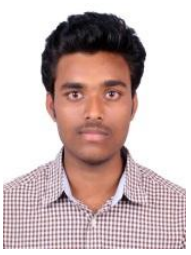

Amol Bhagvat kokare, Student of BE ECE at JSPM's ICOER, Pune.

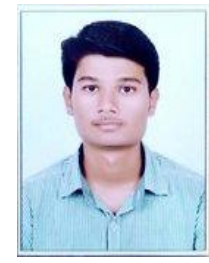

Rohit Gulabrao Aurade, Student of BE ECE at JSPM's ICOER, Pune. 